\title{
Adrenal Venous Sampling: Where Is the Aldosterone Disappearing to?
}

\author{
Miroslav Solar • Jiri Ceral • Antonin Krajina • \\ Marek Ballon · Eva Malirova · Milos Brodak • \\ Jan Cap
}

Received: 28 July 2009/ Accepted: 14 September 2009/Published online: 1 October 2009

(C) The Author(s) 2009. This article is published with open access at Springerlink.com

\begin{abstract}
Adrenal venous sampling (AVS) is generally considered to be the gold standard in distinguishing unilateral and bilateral aldosterone hypersecretion in primary hyperaldosteronism. However, during AVS, we noticed a considerable variability in aldosterone concentrations among samples thought to have come from the right adrenal glands. Some aldosterone concentrations in these samples were even lower than in samples from the inferior vena cava. We hypothesized that the samples with low aldosterone levels were unintentionally taken not from the right adrenal gland, but from hepatic veins. Therefore, we sought to analyze the impact of unintentional cannulation of hepatic veins on AVS. Thirty consecutive patients referred for AVS were enrolled. Hepatic vein sampling was implemented in our standardized AVS protocol. The data were collected and analyzed prospectively. AVS was successful in 27 patients $(90 \%)$, and hepatic vein cannulation
\end{abstract}

M. Solar · J. Ceral $(\bowtie) \cdot$ M. Ballon · J. Cap

Department of Internal Medicine, Charles University Prague, Medical Faculty Hradec Kralove, University Hospital Hradec Kralove, Sokolska 581, 50005 Hradec Kralove, Czech Republic e-mail: ceral.jiri@fnhk.cz

\footnotetext{
A. Krajina

Department of Radiology, Charles University Prague, Medical Faculty Hradec Kralove, University Hospital Hradec Kralove, Sokolska 581, 50005 Hradec Kralove, Czech Republic

E. Malirova

Department of Nuclear Medicine, Charles University Prague, Medical Faculty Hradec Kralove, University Hospital Hradec Kralove, Sokolska 581, 50005 Hradec Kralove, Czech Republic

M. Brodak

Department of Urology, Charles University Prague, Medical Faculty Hradec Kralove, University Hospital Hradec Kralove, Sokolska 581, 50005 Hradec Kralove, Czech Republic
}

was successful in all procedures performed. Cortisol concentrations were not significantly different between the hepatic vein and inferior vena cava samples, but aldosterone concentrations from hepatic venous blood (median, $17 \mathrm{pmol} / \mathrm{l}$; range, 40-860 pmol/l) were markedly lower than in samples from the inferior vena cava (median, $860 \mathrm{pmol} / \mathrm{l}$; range, 460-4510 pmol/l). The observed difference was statistically significant $(P<0.001)$. Aldosterone concentrations in the hepatic veins are significantly lower than in venous blood taken from the inferior vena cava. This finding is important for AVS because hepatic veins can easily be mistaken for adrenal veins as a result of their close anatomic proximity.

Keywords Adrenal venous sampling . Primary aldosteronism

\section{Introduction}

In primary aldosteronism (PA), the choice of treatment depends on the lateralization of aldosterone secretion. In cases with unilateral aldosterone overproduction, adrenalectomy leads to improvement or even a complete cure of high blood pressure. Therefore, it is important to differentiate between bilateral and unilateral forms of PA [1, 2].

The only diagnostic method that can recognize unilateral aldosterone hypersecretion is adrenal venous sampling (AVS) [3, 4]. The adequacy of adrenal blood sampling is evaluated by the ratio of cortisol concentrations in the adrenal vein and in the inferior vena cava $\left(C_{\mathrm{adrenal}} / C_{\mathrm{ivc}}\right)$. The higher the $C_{\text {adrenal }} / C_{\mathrm{ivc}}$ is, the less is the dilution of blood from adrenal veins, which results in a more representative sample of adrenal venous blood. The published criteria for appropriate adrenal vein sampling range from 
Table 1 AVS results of a patient with marked variability in aldosterone concentration in samples thought to come from the right adrenal vein (see samples 2, 3, and 4)

\begin{tabular}{llccrcc}
\hline Sample no. & Sample identification & Aldosterone (pmol/l) & Cortisol (nmol/l) & $C_{\text {adrenal }} / C_{\text {ivc }}$ & $\begin{array}{c}\text { AC } \\
\text { Appropriate } \\
\text { adrenal sampling }^{\text {a }}\end{array}$ \\
\hline 1 & Left adrenal vein & 49,000 & 7,950 & 15.29 & 6.16 & Yes \\
2 & Right adrenal vein & 390 & 930 & 1.79 & 0.42 & No \\
3 & Right adrenal vein & 150 & 680 & 1.31 & 0.22 & No \\
4 & Right adrenal vein & 47,800 & 8,100 & 15.58 & 5.90 & Yes \\
5 & Inferior vena cava $^{b}$ & 1,310 & 520 & & 2.51 & \\
\hline
\end{tabular}

$A V S$ adrenal venous sampling, $A C$ ratio of aldosterone to cortisol concentration, $C_{a d r e n a l} / C_{i v c}$ ratio of the cortisol concentrations in the adrenal vein and the inferior vena cava

${ }^{\text {a }}$ Appropriate adrenal sampling was defined as $C_{\text {adrenal }} / C_{\text {ivc }}$ values of $>5$

b A sample from the inferior vena cava was taken below the renal veins

$C_{\text {adrenal }} / C_{\mathrm{ivc}}$ is $\geq 2-5$ with cosyntropin $[3,5]$ and from $C_{\text {adrenal }} / C_{\text {ivc }}$ is $\geq 1.1-3$ without cosyntropin $[1,6]$.

The ratio of aldosterone to cortisol (AC) is the parameter used to compare both adrenals in order to eliminate variable dilution of adrenal venous blood. The lateralization of aldosterone secretion is assessed by the ratio $\mathrm{AC}_{\mathrm{dominant}}$ adrenal $/ \mathrm{AC}_{\text {nondominant adrenal }}$. The reported criteria for abnormal lateralization of aldosterone secretion also vary significantly, with the ratio $\mathrm{AC}_{\mathrm{dominant}}$ adrenal $\mathrm{AC}_{\text {nondominant adrenal }}$ varying from $\geq 2$ to $\geq 5$ [7]. Moreover, some centers require the suppression of the nondominant adrenal glands $\left(\mathrm{AC}_{\text {nondominant adrenal }}<\mathrm{AC}_{\mathrm{ivc}}\right)$ to confirm abnormal lateralization of aldosterone secretion [8].

When analyzing the AVS results of some patients, we noticed considerable variability in aldosterone concentrations among samples thought to have come from the right adrenal glands. Table 1 presents the AVS data of one such patient as an example. These findings raised doubts about the validity of some of our AVS examinations.

Repeated measurements of hormone concentrations in individual samples excluded laboratory errors. Instead, we hypothesized that some right adrenal samples were actually taken not from adrenal glands but from hepatic veins, which are located nearby. To avoid any further confusion, we implemented hepatic vein sampling into our routine standardized AVS protocol. This approach was chosen primarily to explain the data acquired from individual patients. By using these methods, we were able to systematically analyze the origin of the aldosterone variability among samples thought to come from the right adrenals.

\section{Materials and Methods}

Data from 30 consecutive patients referred to AVS were analyzed. The patients' characteristics are shown in Table 2.
Table 2 Principal characteristics of the study population ${ }^{\mathrm{a}}$

\begin{tabular}{lc}
\hline Characteristic & Value \\
\hline No. of patients (no. women) & $30(4)$ \\
Age (years) & $52(33-71)$ \\
Systolic blood pressure (mmHg) & $133(112-200)$ \\
Diastolic blood pressure (mmHg) & $84(68-110)$ \\
No. of antihypertensive drugs & $4(2-7)$ \\
Serum aldosterone (pmol/l) $^{\mathrm{b}}$ & $510(240-1070)$ \\
${\text { Plasma active renin }(\mathrm{ng} / \mathrm{l})^{\mathrm{b}}}^{\text {Aldosterone to renin ratio }}$ & $2.10(0.48-7.15)$ \\
\hline
\end{tabular}

$\overline{\text { a Values are expressed as median (range) unless otherwise indicated }}$

b Screening values

c Ratio of aldosterone to renin concentration was calculated in conventional units (ng/l)

Indication of AVS resulted from a high clinical suspicion of PA, which was based on a previous noninvasive diagnostic workup. Withdrawal of aldosterone antagonists for 2 months was required before the procedures were initiated. The ratio of aldosterone to active renin concentration (ARR) was used to screen patients for PA. Blood sampling for ARR was always performed in the morning hours (7-10 a.m.) with the patients in the upright position for at least $30 \mathrm{~min}$ before sample collection. For calculation of ARR, both serum aldosterone and plasma renin concentrations were expressed in ng/l. ARR values of $>20$ were considered to be abnormal, and on the basis of our observations in 69 healthy volunteers (unpublished data), 20 was set as the cutoff value. Before sampling for ARR, no specific changes in antihypertensive medication were initially required (except for the withdrawal of any aldosterone antagonists as mentioned above).

However, if the patient had an ARR of $>20$, adrenal adenoma, or hypokalemia, ARR sampling was repeated after 2 weeks of withdrawal from any medication that could interfere with the renin-angiotensin-aldosterone system. 
Only doxazosin and verapamil were allowed for patients who were considered at risk from uncontrolled hypertension.

On the basis of persistent increase of ARR $(>20)$, the patients were referred for suppression testing. Verapamil and doxazosin were the only antihypertensive drugs allowed. Blood samples for serum aldosterone were taken in the supine position after a 4-h saline infusion $(2000 \mathrm{ml})$ and a previous period of high salt intake. Aldosterone was considered nonsuppressible when it was $>100 \mathrm{pmol} / \mathrm{l}$, which, on the basis of our observations from 32 healthy volunteers (unpublished data), was the cutoff value.

All AVS procedures were performed according to the routine standardized protocol used in our center. No changes in concomitant medications were required, except for oral anticoagulants and aldosterone antagonists that had to be withdrawn before the procedure. All of the patients provided informed written consent for the AVS procedure according to the following protocol. To minimize stressinduced fluctuations in adrenal hormones secretion, an infusion of cosyntropin was administered $1 \mathrm{~h}$ before and during the procedure, at the rate of $160 \mu \mathrm{g} / \mathrm{h}$. The design of the cosyntropin dosing scheme was inspired by previous reports $[9,10]$.

Samples were taken step by step from both adrenal veins, one of the hepatic veins, and the inferior vena cava below the renal veins $[11,12]$. Because cannulation of adrenal veins, especially the right one, is technically difficult, multiple samples were taken in order to increase the success rate of the procedure.

Angiographic verification of the catheter placement into adrenal and hepatic veins was required both before and just after the sampling in order to exclude the possibility of catheter displacement during blood sampling [12]. Small amounts of contrast agent were used to minimize the risk of adrenal injury.

Aldosterone and cortisol concentrations were measured in all the acquired samples. Commercially available radioimmunoassay kits were used both to measure aldosterone (Diagnostic Products Corporation, Los Angeles, CA) and cortisol (Immunotech, Beckman Coulter, Marseille, France). In the long term, the observed intra-assay coefficients of variation were less than $7 \%$ for aldosterone and no more than $5 \%$ for cortisol. The cross-reactivity of the diagnostic antibodies for other corticosteroids was low.

AVS was considered successful when samples with $C_{\text {adrenal }} / C_{\text {ivc }}$ values of $>5$ were obtained from both adrenals. Abnormal lateralization of aldosterone secretion was defined by $\mathrm{AC}_{\text {dominant adrenal }} / \mathrm{AC}_{\text {nondominant adrenal values of }}$ $>4$ [5]. These criteria were chosen to avoid uncertainties when indicating adrenalectomy.

All the patients provided written informed consent to the AVS procedure according to the above-mentioned protocol. Institutional review board approval was not required.
Nonparametric tests were used for statistical analysis of the acquired data (MedCalc, Mariakerke, Belgium).

\section{Results}

The AVS procedure was successful in 27 patients $(90 \%)$ when samples with a $C_{\text {adrenal }} / C_{\mathrm{ivc}}$ value of $>5$ were obtained from both adrenals. One patient was catheterized twice because the first catheterization failed to yield adequate samples. No complications were noted. By using samples with the highest $C_{\text {adrenal }} / C_{\mathrm{ivc}}$, a lateralization of aldosterone secretion $\left(\mathrm{AC}_{\mathrm{dominant} \text { adrenal }} / \mathrm{AC}_{\text {nondominant adrenal }}\right.$ >4) was diagnosed in eight cases (26.7\%).

Hepatic vein cannulation was successful in all AVS procedures performed. The aldosterone concentration was uniformly lower in hepatic veins than in the inferior vena cava. The medians of aldosterone concentrations in hepatic venous blood and the inferior vena cava were 170 (range, 40-860) pmol/l and 860 (range, 460-4510) pmol/l, respectively (Fig. 1). The observed difference was highly statistically significant $(P<0.001)$ by the Wilcoxon test.

In contrast, no significant difference in the cortisol concentrations was observed between hepatic veins (median, $810 \mathrm{nmol} / \mathrm{l}$; range, $510-1350 \mathrm{nmol} / \mathrm{l}$ ) and inferior vena cava (median, $790 \mathrm{nmol} / \mathrm{l}$; range, 540-1 $500 \mathrm{nmol} / \mathrm{l}$ ).

In patients with successful AVS, the ratio of cortisol concentrations in the hepatic vein and inferior vena cava $\left(C_{\text {hepatic }} / C_{\mathrm{ivc}}\right)$ was calculated as a parameter, in a manner analogous to $C_{\text {adrenal }} / C_{\mathrm{ivc}}$. Marked variability in $C_{\mathrm{hepatic}} / C_{\mathrm{ivc}}$ was noted (range, 0.74-2). In eight patients (29.6\%), the $C_{\text {hepatic }} / C_{\mathrm{ivc}}$ value was $\leq 0.9$, and in seven patients (25.9\%), the $C_{\text {hepatic }} / C_{\mathrm{ivc}}$ value was $\geq 1.1$.

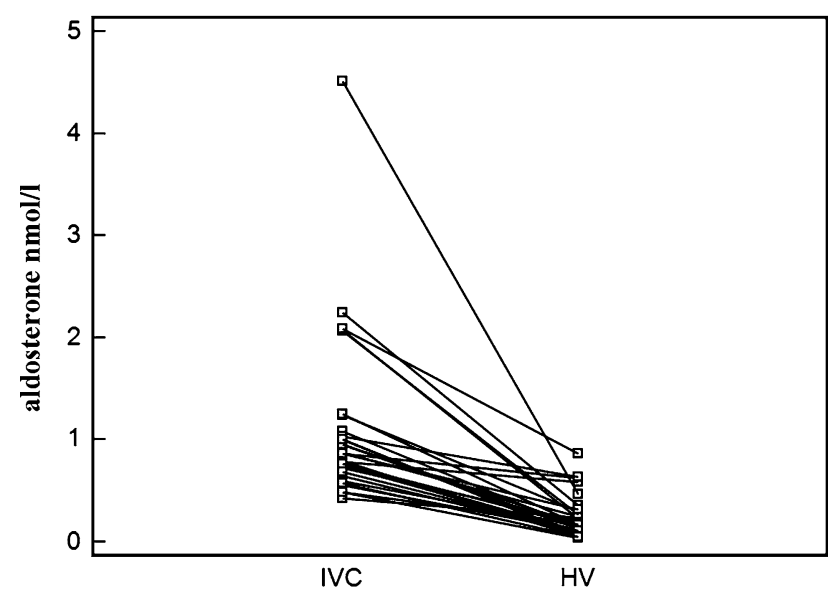

Fig. 1 Aldosterone concentrations in the HV and in the IVC. Graph compares the aldosterone concentrations in the $\mathrm{HV}$ and in the IVC. Aldosterone concentration in HV was uniformly and significantly $(P$ $<0.001)$ lower than in the IVC. $H V$ hepatic vein, $I V C$ inferior vena cava 
If hepatic samples with $C_{\text {hepatic }} / C_{\text {ivc }}$ values of $\geq 1.1$ had been considered to be from the right adrenal, the lowest published criterion for appropriate AVS [6] would have been satisfied.

Replacing the right adrenal samples with the corresponding hepatic samples with $C_{\text {hepatic }} / C_{\text {ivc }}$ values of $\geq 1.1$ would have led to changes in the AVS interpretation in all seven patients. In six patients without lateralization of aldosterone secretion, false hypersecretion from the left adrenal would have been detected. Moreover, in one patient with right-side aldosterone overproduction, accidental hepatic vein sampling would have masked the lateralization of aldosterone secretion.

\section{Discussion}

Implementation of hepatic sampling into our routine AVS protocol cleared up some of our doubts about the clinical interpretation of the data acquired. By taking one additional control sample from the hepatic veins during AVS, we were able to explain the observed discrepancies in aldosterone concentrations among "right adrenal" venous samples in individual patients.

When we analyzed the data from hepatic samples, we observed that liver metabolism of aldosterone exceeded that of cortisol. Aldosterone concentrations found in the hepatic venous blood were only a fraction of those found in the inferior vena cava, whereas cortisol levels were not significantly different. The high metabolic rate of aldosterone in the liver is well known [13, 14]. However, to our knowledge, it has not been mentioned in the context of AVS. In particular, a low aldosterone concentration in the hepatic venous blood may have implications for AVS interpretation if a sample of hepatic venous blood is mistakenly thought to come from the right adrenal vein. Cortisol concentration does not enable the differentiation of adrenal and nonadrenal samples when the lowest recommended $C_{\text {adrenal }} / C_{\text {ivc }}$ cutoff criterion [15] is used. In a quarter of the examined patients, the estimated cortisol concentration in the liver was at least $10 \%$ higher than that in the inferior vena cava. In our opinion, there are several reasons that may explain this finding.

First, the most obvious explanation seems to be the inherent imprecision of laboratory assays of cortisol. The precision of a laboratory test describes a coefficient of variation that is defined by the ratio of the standard deviation (SD) to the mean. According to statistics, $68.3 \%$ of values measured from one sample are within the range of one SD from the mean, and $99.7 \%$ of measured values are within the range of 3 SDs. Therefore, when the coefficient of variation of the cortisol laboratory assessment is $5 \%, 31.7 \%$ of the measured values (from one sample) lie more than $\pm 5 \%$ away from the mean. Consequently, it is not unlikely that the estimated cortisol concentrations in two nonadrenal samples could differ by more than $10 \%$. Second, despite continuous cosyntropin infusion during sampling, we remained unable to exclude small fluctuations in serum cortisol. These arguments support the fact that the number of hepatic samples with $C_{\text {hepatic }} / C_{\text {ivc }}$ values of $\geq 1.1(25.9 \%)$ corresponds to the proportion of examinations $(29.6 \%)$ with $C_{\text {hepatic }} / C_{\mathrm{ivc}}$ values of $\leq 0.9$.

Adrenal samples containing a significant portion of nonadrenal blood can influence the results, especially when they are contaminated with hepatic venous blood, which has a low concentration of aldosterone. As a potential cause, the anomalous confluence of adrenal and hepatic vein has been described [16]. However, the frequency and the clinical significance of this anomaly are poorly understood [17, 18]. In our study, we did not systematically assess adrenal venous anatomy. Retrograde venography with a large volume of contrast agent would greatly increase the risk of adrenal hemorrhage. Moreover, venography itself cannot depict related parenchymatous organs or differentiate anomalous hepatic veins from right adrenal veins [19].

In practice, displacement of the catheter during sampling can occur. High concentrations of adrenal hormones can be found in the blood taken from the inferior vena cava above the adrenal veins [18] because the laminar blood flow may prevent complete dilution of blood coming from adrenal veins. However, this may also be true for blood with a low concentration of aldosterone coming from hepatic veins lying close to the adrenal veins.

We tried to eliminate this effect by performing adrenal angiography not only before but also after sampling, but adherence to this rule was not absolute. As an example, we present AVS data of one patient in whom it was difficult to cannulate a right adrenal vein (Table 3 ). Inconsistently with our protocol, not all samples were taken under predefined angiographic guidance. Cortisol-corrected aldosterone concentrations (AC) differed greatly between samples with moderate and high $C_{\text {adrenal }} / C_{\mathrm{ivc}}$ values. Moreover, marked AC differences were observed among samples with moderate $C_{\text {adrenal }} / C_{\text {ivc }}$ values (Table 3 , samples $2,6,7$, and 8 ), which can be explained by variable dilution of adrenal venous blood. In fact, as demonstrated in Table 3, two samples ascribed to right adrenal veins were taken not from the adrenal but from the liver (Table 3, samples 3 and 4).

Therefore, the only principal determinant of successful adrenal sampling is a high concentration of cortisol. The recent guidelines on $\mathrm{PA}$ [20] report an adrenal/peripheral vein cortisol ratio that is typically more than $10: 1$ with the continuous cosyntropin infusion protocol, and more than 
Table 3 AVS results of one patient in whom it was difficult to cannulate a right adrenal vein ${ }^{\mathrm{a}}$

\begin{tabular}{|c|c|c|c|c|c|c|}
\hline Sample no. & Sample identification & $\begin{array}{l}\text { Aldosterone } \\
(\mathrm{pmol} / \mathrm{l})\end{array}$ & $\begin{array}{l}\text { Cortisol } \\
(\mathrm{nmol} / \mathrm{l})\end{array}$ & $C_{\text {adrenal }} / C_{\mathrm{ivc}}$ & $\mathrm{AC}$ & $\begin{array}{l}\text { Appropriate } \\
\text { adrenal sampling }\end{array}$ \\
\hline 1 & Left adrenal vein & 87,100 & 23,500 & 25.54 & 3.71 & Yes \\
\hline 2 & Right adrenal vein & 12,100 & 3,800 & 4.13 & 3.18 & No \\
\hline 3 & Right adrenal vein & 470 & 920 & 1.00 & 0.51 & No \\
\hline 4 & Right adrenal vein & 360 & 800 & 0.87 & 0.45 & No \\
\hline 5 & Right adrenal vein & 101,600 & 26,700 & 29.02 & 3.81 & Yes \\
\hline 6 & Right adrenal vein & 4,400 & 2,800 & 3.04 & 1.57 & No \\
\hline 7 & Right adrenal vein & 2,010 & 3,100 & 3.37 & 0.65 & No \\
\hline 8 & Right adrenal vein & 7,400 & 2,800 & 3.04 & 2.64 & No \\
\hline 9 & Hepatic vein & 270 & 970 & $1.05^{\mathrm{c}}$ & 0.28 & \\
\hline 10 & Inferior vena cava $^{\mathrm{d}}$ & 2,070 & 920 & & 2.25 & \\
\hline
\end{tabular}

$A V S$ adrenal venous sampling, $A C$ ratio of aldosterone to cortisol concentration, $C_{a d r e n a l} / C_{i v c}$ ratio of the cortisol concentrations in the adrenal vein and the inferior vena cava

${ }^{\text {a }}$ Cortisol-corrected aldosterone concentrations (AC) differed greatly between samples with moderate or high $C_{\text {adrenal }} / C_{\mathrm{ivc} .}$ Marked $\mathrm{AC}$ variability was observed among samples with moderate $C_{\text {adrenal }} / C_{\mathrm{ivc}}$ (samples $2,6,7$, and 8). Samples 3 and 4 were unintentionally taken not from the right adrenal but from the liver; their aldosterone concentrations are markedly lower than those of samples from the inferior vena cava and from the right adrenal vein

${ }^{\mathrm{b}}$ Appropriate adrenal sampling was defined by $C_{\text {adrenal }} / C_{\mathrm{ivc}}>5$

${ }^{\mathrm{c}} C_{\text {hepatic }} / C_{\mathrm{ivc}}$

d A sample from the inferior vena cava was taken below the renal veins

3:1 without the use of cosyntropin. In fact, the published criteria for adequate adrenal sampling, with or without adrenal stimulation, are based primarily on local experience and personal opinions. They vary greatly from $C_{\text {adre- }}$ nal $/ C_{\mathrm{ivc}}$ values of $\geq 1.1$ [6] to $C_{\text {adrenal }} / C_{\mathrm{ivc}}$ values of $>5$ [5].

It is unclear to what extent our findings are applicable to AVS procedures performed without adrenal stimulation $[21,22]$. However, our data clearly show that appropriate choice of a $C_{\text {adrenal }} / C_{\mathrm{ivc}}$ cutoff should at least eliminate the inherent imprecision of cortisol laboratory estimation.

To conclude, we found that aldosterone concentration in hepatic veins is uniformly lower than in mixed venous blood taken from the inferior vena cava; however, no difference in cortisol concentrations was observed between the two veins. This finding may have implications for AVS because unintentional cannulation of hepatic veins instead of right adrenal veins can occur during adrenal catheterization. Caution should be taken when samples characterized by only mild increase in cortisol concentration (compared with inferior vena cava) are used for the interpretation of the results.

Acknowledgment This work was supported as part of research projects MZO00179906 and MSM0021620817.

Open Access This article is distributed under the terms of the Creative Commons Attribution Noncommercial License which permits any noncommercial use, distribution, and reproduction in any medium, provided the original author(s) and source are credited.

\section{References}

1. Stowasser M, Gordon RD (2003) Primary aldosteronism. Best Pract Res Clin Endocrinol Metab 17:591-605

2. Young WF (2007) Primary aldosteronism: renaissance of a syndrome. Clin Endocrinol (Oxf) 66:607-618

3. Mulatero P, Bertello C, Rossato D et al (2008) Roles of clinical criteria, computed tomography scan, and adrenal vein sampling in differential diagnosis of primary aldosteronism subtypes. J Clin Endocrinol Metab 93:1366-1371

4. Young WF, Stanson AW (2009) What are the keys to successful adrenal venous sampling (AVS) in patients with primary aldosteronism? Clin Endocrinol (Oxf) 70:14-17

5. Young WF, Stanson AW, Thompson GB et al (2004) Role for adrenal venous sampling in primary aldosteronism. Surgery 136 : $1227-1235$

6. Rossi GP, Sacchetto A, Chiesura-Corona M et al (2001) Identification of the etiology of primary aldosteronism with adrenal vein sampling in patients with equivocal computed tomography and magnetic resonance findings: results in 104 consecutive cases. J Clin Endocrinol Metab 86:1083-1090

7. Mulatero P, Stowasser M, Loh KC et al (2004) Increased diagnosis of primary aldosteronism, including surgically correctable forms, in centers from five continents. J Clin Endocrinol Metab 89:10451050

8. Stowasser M, Gordon RD (2004) Primary aldosteronism—careful investigation is essential and rewarding. Mol Cell Endocrinol 217:33-39

9. Doppman JL, Gill JR Jr, Miller DL et al (1992) Distinction between hyperaldosteronism due to bilateral hyperplasia and unilateral aldosteronoma: reliability of CT. Radiology 184:677-682

10. Weinberger MH, Grim CE, Hollifield JW et al (1979) Primary aldosteronism: diagnosis, localization, and treatment. Ann Intern Med 90:386-395 
11. Young WF Jr, Stanson AW, Grant CS et al (1996) Primary aldosteronism: adrenal venous sampling. Surgery 120:913-919

12. Magill SB (2001) Adrenal vein sampling: an oveview. Endocrinologist 11:357-363

13. Coppage WS Jr, Island DP, Cooner AE et al (1962) The metabolism of aldosterone in normal subjects and in patients with hepatic cirrhosis. J Clin Invest 41:1672-1680

14. Luetscher JA, Hancock EW, Camargo CA et al (1965) Conjugation of 1,2-3H-aldosterone in human liver and kidneys and renal extraction of aldosterone and labeled conjugates from blood plasma. J Clin Endocrinol Metab 25:628-638

15. Rossi GP, Pitter G, Bernante P et al (2008) Adrenal vein sampling for primary aldosteronism: the assessment of selectivity and lateralization of aldosterone excess baseline and after adrenocorticotropic hormone (ACTH) stimulation. J Hypertens 26:989-997

16. MacGillivray DC, Khwaja K, Shickman SJ (1996) Confluence of the right adrenal vein with the accessory right hepatic veins. A potential hazard in laparoscopic right adrenalectomy. Surg Endosc 10:1095-1096
17. Sebe P, Peyromaure M, Raynaud A et al (2002) Anatomical variations in the drainage of the principal adrenal veins: the results of 88 venograms. Surg Radiol Anat 24:222-225

18. Daunt N (2005) Adrenal vein sampling: how to make it quick, easy, and successful. Radiographics 25(suppl 1):S143-S158

19. Lau JH, Drake W, Matson M (2007) The current role of venous sampling in the localization of endocrine disease. Cardiovasc Intervent Radiol 30:555-570

20. Funder JW, Carey RM, Fardella C et al (2008) Case detection, diagnosis, and treatment of patients with primary aldosteronism: an endocrine society clinical practice guideline. J Clin Endocrinol Metab 93:3266-3281

21. Magill SB, Raff H, Shaker JL et al (2001) Comparison of adrenal vein sampling and computed tomography in the differentiation of primary aldosteronism. J Clin Endocrinol Metab 86:1066-1071

22. Rossi GP, Ganzaroli C, Miotto D et al (2006) Dynamic testing with high-dose adrenocorticotrophic hormone does not improve lateralization of aldosterone oversecretion in primary aldosteronism patients. J Hypertens 24:371-379 